\title{
Initial Development of a PDA Mobility Aid for Visually Impaired People
}

\author{
David McGookin ${ }^{1}$, Maya Gibbs ${ }^{1}$, Annu-Maaria Nivala ${ }^{2}$, and Stephen Brewster ${ }^{1}$ \\ ${ }^{1}$ Department of Computing Science, University of Glasgow, Glasgow G12 8QQ, UK \\ \{mcgookdk, stephen\}@dcs.gla.ac.uk \\ ${ }^{2}$ Finnish Geodetic Institute, Geodeetinrinne 2, P.O. Box 15, Finland \\ annu-maaria.nivala@fgi.fi
}

\begin{abstract}
We discuss requirements surrounding a mobile navigation system for visually impaired people. We describe an initial prototype based on a PDA using GPS location tracking. This prototype has so far failed to provide reliable location detection, due to the use of GPS in built up environments. We discuss how our system may improve detection by switching between a range of different location tracking technologies. However, we conclude that there may still be times when these technologies fail, and more work is needed on how to support the user in such circumstances.
\end{abstract}

\section{Introduction}

When navigating an environment, visually impaired people are presented with a number of problems. Hazard avoidance such as street furniture and uneven surfaces can be overcome with the use of a white cane or guide dog. However "macro-navigation", finding directions from A to B [1], presents more problems. Many local government organisations provide mobility officers who can help a visually impaired person navigate the environment, and help with learning (or re-learning) locations. However, such services are limited, and not of use for people visiting other towns or places when on business or holidays. A way to overcome these problems would be the use of a mobile navigation device, which would help to guide a visually impaired person around an environment. Several such systems have been proposed, but few evaluated. The most notable was MoBIC developed by Petrie et al. 11. Further research has been carried out since then, but mostly on different tracking systems and alternate interfaces to display information to the user 2]. Whilst these are important, in the last few years the growth in availability and power of mobile devices, as well as increasing mobile availability of the internet, has increased the potential functionality of mobile navigation systems for the visually impaired. We believe it is therefore appropriate to investigate mobile navigation systems on low cost mobile devices. In this paper we present our initial requirements capture and prototype system, as well as issues for future design decisions. 


\section{Issues Surrounding Navigation}

To further understand the problems of navigation for visually impaired people, and determine requirements for our system, we carried out a semi-structured interview with a middle aged, blind individual who worked as a transcription officer for the Royal National Institute of the Blind (RNIB) in the UK. The interview covered navigation in the world and how this was accomplished.

An emphasis on macro rather than micro navigation was emphasised. The use of a white cane to detect immediate obstacles was successful, but the ability to navigate to and from unfamiliar locations was more difficult. As the participant noted, "I certainly wouldn't turn up at, say, Aberdeen (railway) station and expect to navigate my way somewhere. Even if it is only within 200 yards.". The participant also noted than in addition to just directions from A to B, information about temporary impediments to navigation would be useful. These seem to fall somewhat between micro and macro navigation, being things which would be detectable with a white cane aid, but would be beneficial to avoid all together. For example, when a pavement (sidewalk) is being resurfaced and pedestrians are diverted through a sectioned off area of the road. Additionally, he noted that it would be useful to select routes on more that simply shortest distance, with other factors such as avoiding traffic or steep inclines being preferred. The participant felt that people "don't generally like looking out of place, or sounding out of place", so the system should not draw attention to the user. Therefore speech input was felt to be inappropriate, but speech output was preferred as walking using headphones "doesn't look too out of place these days".

The requirements identified here are broadly in line with those identified by Strothotte et al. [3] as part of to MoBIC project, highlighting that users still have the same basic needs although technology has moved on in the last decade.

\section{Prototype Overview}

Based on the requirements identified, we designed and implemented an initial prototype to provide route navigation abilities. Once the initial capabilities have been implemented and evaluated, we will be able to implement more of the features identified during our requirements capture.

Our initial system runs as an application on a Dell Axim Pocket PC, and retrieves location information using the global positioning system (GPS) via a TomTom Bluetooth GPS receiver. At the moment the application automatically detects the user's location and then calculates a route to a destination based on selection from a list. We store data in files formatted in Geographic Mark-up Language which is a standard XML compliant format developed for cartographic information. Once loaded into our system the map is held as a weighted graph (each road segment is assigned a "weight" dependent on its length), and the route to the user's destination is calculated using a shortest path algorithm. That is to say that the application calculates the route with the lowest sum total of weights. Whilst in our initial system weightings are strictly geographic 
distances, we can easily change the weightings to reflect different navigation priorities, such as avoiding particularly steep or busy roads. In the future we may be able to interface the system, through wireless networks, to local council works notifications to bias against areas where road works or other construction is occurring, thereby addressing some of the issues identified in our interview.

As was requested in the requirements phase, synthetic speech was used to provide all feedback from the system. Once the user has selected a destination, the system informs what direction to walk in, and then when to turn left or right at appropriate intersections. The user can also request the system provides the current location. Output is played through a single ear piece or, if the user prefers, the PDA's in-built speaker.

To simplify user input we have applied a simple menu based interface. A back, forward and select button are presented on the PDA screen and can be felt by the user via a raised paper control panel overlaying the touch screen. This allows controls to be shaped differently and therefore be easier to identify than using the inbuilt buttons on the PDA, or using the keypad of a smart phone device. However we must still identify how well this interface will scale when more functions are added.

\section{Initial Evaluations}

So far we have not carried out any field trials of our system using visually impaired people. We have however carried out technical evaluations to establish the quality of information available from our system, and a short user evaluation of the interface. Whilst users found the interface straightforward to navigate, the location detection of our system has been less successful. Our system, in a desire to use "off the shelf" location tracking, uses standard GPS. In our tests this has proven to be an inadequate technology to support the navigation tasks, with many occasions where the system failed to correctly detect its position and inform the user when to change direction. That GPS is poor in built up environments is a known problem (our test area was on a hill surrounded by tall buildings), and many others have proposed using differential GPS [2] which uses an additional ground transmitter to improve location. However these systems still require line of sight with GPS satellites, otherwise incorrect locations will be determined. For example, the MoBIC system was evaluated in an area with open spaces [1]. However many towns and cities do not always afford such open space. Other systems propose that the environment be augmented with radio frequency identification (RFID) tags to more easily detect location [4, but these must be available across the whole area to be useful. Another technology, not yet applied to visually impaired navigation, is Skyhook Technology's Loki system (www.skyhookwireless.com). This uses an updated map of available Wi-Fi access points to determine the location of the user. However accurate location detection is currently only available in a limited number of locations. There is not currently a single technology that can always be relied upon to deliver accurate positioning. 


\section{Future Work}

Whilst it may be possible to use different technology to determine the user's location, these different technologies produce that information with differing degrees of fidelity. When comparing the position of multiple technologies there may be conflicts between their reported positions, or in some cases, no position at all. Whilst we will seek to improve the accuracy of detecting the user's location by using different, and perhaps multiple technologies, we also need to determine what should happen when an accurate position cannot be determined. The variable coverage of location based technologies is currently being studied in visual scenarios as seamful design [5]. Here the breaks in technologies, the seams, rather than being hidden from the user, are made more obvious, allowing awareness of problems before they occur. For example, our navigation system may monitor areas of poor signal strength and notify the user when it detects that it is at the boundary of that area. Further investigation into the exposition of seams in such non-visual applications may help to overcome many of the problems of currently tracking the position of the user.

\section{Conclusions}

Mobile navigation presents a significant problem for visually impaired people. From our requirements capture we can identify several issues that could be overcome with the constructive use of technology. However, from our initial prototype system, there are several problems in the use of tracking technology that can easily cause the user's location to be mistakenly detected. Whilst we believe that the position tracking of our system can be improved, there remains no one technique that can guarantee correct location, and any system will, at times, lose the position of the user. We propose that further research, on how best to make the user aware of failings in tracking technology in non-visual scenarios would significantly improve the quality of mobile navigation solutions.

\section{References}

1. Petrie, H., Johnson, V., Furner, S., Strothotte, T.: Design lifecycles and wearable computers for users with disabilities. In: Proceedings of the first international workshop of Human Computer Interaction with mobile devices, Glasgow, UK, GIST, vol. 1 (1998)

2. Loomis, J.M., Klatzky, R.L., Colledge, R.G.: Navigating without vision: Basic and applied research. Optometry \& Vision Science 78(5), 282-289 (2001)

3. Strothotte, T., Petrie, H., Johnson, V., Reichert, L.: Mobic: User needs and preliminary design for mobility aid for blind and elderly travellers. In: Placencia Porrero, I., Puig de la Bellacasa, R. (eds.) The European Context for Assistive Technology, pp. 384-352. IOS Press, Amsterdam (1995)

4. Coroama, V.: Experiences from the design of a ubiquitous computing system for the blind. In: CHI 2006, Montreal, Canada, vol. 2, pp. 664-669. ACM Press, New York (2006)

5. Chalmers, M., Dieberger, A., Höök, K., Rudström, A..: Social navigation and seamful design. Cognitive Studies 11(3), 1-11 (2004) 